\title{
Influência da dor crônica na capacidade funcional de idosos institucionalizados
}

\author{
Influence of chronic pain in the functional capacity of institucionalized elderly
}

Influencia del dolor crónico en la capacidad funcional de ancianos internados en instituciones

\author{
Luciana Araújo Reis', Gilson de Vasconcelos Torres \\ 'Universidade Federal do Rio Grande do Norte. Natal RN
}

Submissão: 05/01/2010

Aprovação: 20/1 1/2010

\section{RESUMO}

Este estudo teve por objetivo analisar a influência da dor Quanto à duração, localização e intensidade na capacidade funcional de idosos institucionalizados. Trata-se de um estudo tansversal a partir de uma amostra de 60 idosos. O instrumento foi composto por variáveis sociodemográficas, de saúde, escala numérica de dor e Índice de Barthel. Com a aplicação do Teste Qui-Quadrado verificou-se diferença estatística significante entre presença de dor e as atividades: banho $(p=0,015)$ vestir-se $(p=0,041)$, transferência para higiene intima $(p=0,00 \mathrm{I})$, transferência cama e cadeira $(p=0,032)$, deambulação $(p=0,010)$ e subir escadas $(p=0,008)$ e a pontuação do Índice de Barthel e presença de dor, $p<0,000$. Diante dos resultados deste estudo constatou-se Que a dor interfere de maneira negativa na capacidade funcional dos idosos.

Descritores: Idoso; Dor; Atividades cotidianas.

\section{ABSTRACT}

The aim of this study was to analyze the influence of pain, in terms of its duration, location and intensity, on the functional capacity of institutionalized elderly. That was a cross-sectional study carried out with a sample of 60 elderly patients. The instrument was composed by sociodemographic and health variables, the numerical pain scale and the Barthel scale. The Chi-square test showed a significant statistical difference between the presence of pain and the following activities: bathing $(p=0.015)$ dressing $(p=0.041)$, toilet use $(p=0.001)$, bed-chair transfer $(p=0.032)$, ambulation $(p=0.010)$ and stairs climbing $(p=0.008)$ and between total Barthel score and the presence of pain, $p<0,000$. The results of this study show that pain has a negative effect on the functional capacity of the elderly.

Descriptors: Elderly; Pain; Activities of daily living.

\section{RESUMEN}

Este estudio tuvo como objetivo analizar la influencia del dolor cuanto a su duración, localización e intensidad en la capacidad funcional de ancianos internados en instituciones. Se trata de un estudio descriptivo con delineación transversal y una muestra de 60 ancianos. El instrumento se basa en variables sociodemográficas, de salud, escala numérica de dolor e Índice de Barthel. Con la aplicación del Test Chi-cuadrado se verificó Que hay diferencia estadística significativa entre la presencia de dolor en las actividades: baño $(p=0,015)$ vestirse $(p=0,041)$, traslado para labores de higiene intima $(p=0,001)$, traspaso a cama y silla $(p=0,032)$, locomoción $(p=0,0$ I 0$)$ y subir escaleras $(p=0,008)$ y entre la puntuación total de Barthel y presencia de dolor, $p<0,000$. A la vista de los resultados de este estudio se constata Que el dolor interfiere de manera negativa en la capacidad funcional de los ancianos.

Descriptores: Anciano; Dolor; Actividades cotidianas.

AUTOR CORRESPONDENTE Gilson de Vasconcelos Torres. Universidade Federal do Rio Grande do Norte. Caixa Postal I524. CEP 59072-970.

Natal, RN. E-mail: gtv@ufrnnet.br 


\section{INTRODUÇÃO}

O crescente processo de envelhecimento populacional ocorrido inicialmente nos chamados países de primeiro mundo tem atingindo também os países em processo de desenvolvimento, como é o caso do Brasil. Tal processo tende a provocar alterações sociais, econômicas e de saúde, gerando novas demandas para o setor público, sobressaindo-se os cuidados de longa duração dirigidos a idosos com algum grau de dificuldade para a execução das atividades da vida diária e/ou aqueles cuja família não possui meios financeiros, físicos ou emocionais para a prestação dos cuidados necessários.

À medida Que a idade aumenta o indivíduo progressivamente tende a se sentir fragilizado e desamparado, não só diante da família, mas de toda sociedade. Sendo assim tem sido visto como improdutivo e nem sempre é acolhido pela família, e são obrigados a morar em asilos ou albergues ${ }^{(1)}$. Os asilos são geralmente locais inapropriados e inadequados às necessidades do idoso, vindo a dificultar as relações interpessoais indispensáveis à manutenção do idoso pela vida e pela construção da cidadania ${ }^{(2)}$. Além disso, tendem a promover seu isolamento, sua inatividade física e mental, tendo dessa forma, consequências negativas à sua saúde ${ }^{(3)}$.

$\mathrm{O}$ processo de envelhecimento, na maioria das vezes, não se caracteriza como um período saudável e de independência. Ao contrário, caracteriza-se pela alta incidência de doenças crônicas e degenerativas Que, muitas vezes, resultam em elevada dependência. Muito desses Quadros são acompanhados por dor e, em significativa parcela deles, a dor crônica é a principal Queixa do indivíduo, fato Que pode interferir de modo acentuado na Qualidade de vida dos idosos ${ }^{(4)}$.

As incapacidades física, psicológica e social são as principais repercussões Que acometem a Qualidade de vida dos idosos com dor crônica, sendo importante a sua identificação ${ }^{(5)}$. Estudos têm demonstrado Que atividades funcionais da vida diária podem ser mais sensíveis às alterações dolorosas ${ }^{(5-6)}$.

A capacidade do indivíduo realizar suas atividades físicas e mentais necessárias para a manutenção de suas atividades básicas e instrumentais, ou seja: tomar banho, vestir-se, realizar higiene pessoal, transferir-se, manter a continência, preparar refeições, controle financeiro, tomar remédios, arrumar a casa, fazer compras, usar transporte coletivo, usar telefone e caminhar certa distância são definidos como capacidade funcional ${ }^{(7-8)}$.

A análise da funcionalidade consiste na mensuração e na classificação da capacidade funcional, para Que se identifieuem as suas limitações $^{(8)}$. Para isso, utiliza-se uma avaliação com base na capacidade de execução das atividades da vida diária, através de medidas indiretas de escalas como o Índice de Barthel, o Índice de Katz, entre outros ${ }^{(9)}$.

Neste estudo optou-se por avaliar as atividades de vida diária, sendo adotado para tanto o Índice de Barthel. Segundo estudos realizados com a aplicação do Índice de Barthel, a cada ano, cerca de $10 \%$ da população adulta, a partir dos 75 anos perde a independência em uma ou mais atividades básicas da vida diária, tais como: banhar-se, vestir-se, alimentar-se e higiene pessoal ${ }^{(9)}$.

Nesta perspectiva, considerando Que a presença de dor no idoso constitui um problema de relevância no contexto atual social e de saúde, principalmente Quando esta influencia na limitação da ca- pacidade funcional, temos como objeto de estudo a alteração da capacidade funcional do idoso asilado decorrente da dor.

A perda da capacidade funcional está associada à predisposição de fragilidade, dependência, institucionalização, risco aumentado de Quedas, morte e problemas de mobilidade, trazendo complicações ao longo do tempo, e gerando cuidados de longa permanência e alto custo ${ }^{(10)}$.

Nesta perspectiva, este estudo tem por objetivo analisar a influência da dor euanto à duração, localização e intensidade na capacidade funcional de idosos institucionalizados.

\section{MÉTODOS}

Trata-se de uma pesquisa com delineamento transversal. O local de estudo foi a Fundação Leur Brito, única instituição de acolhimento exclusivo de idosos, no município de Jequié/BA. A população alvo do estudo foi composta por todos os idosos residentes na instituição asilar já referida no período proposto para coleta de dados. Sendo o critério de exclusão, idosos Que não tivessem condições mentais para responder ao roteiro de entrevista e Que não Quisessem participar voluntariamente do estudo.

Para avaliar o estado mental (condições cognitivas) dos idosos foi utilizado o Mini-exame do estado mental - MEEM (Mini-mental). Do total de 68 idosos residentes no asilo oito foram excluídos por apresentarem escore do MEEM inferior a 23 pontos (sugestivo de déficit cognitivo) e incluídos 60 idosos, sendo 30 de cada sexo, $40 \%$ apresentavam funções cognitivas preservadas e $60 \%$ demonstravam alguma alteração cognitiva não sugestiva de déficit.

O instrumento de pesquisa utilizado para a coleta de dados neste estudo foi um formulário estruturado de entrevista constituído de Quatro partes: 1) Caracterização sóciodemográfica e de saúde; 2) Aspectos relacionados a dor (tempo e localização); 3) Avaliação da dor pela Escala Numérica e 4) Capacidade funcional medida pelo Índice de Barthel.

A Escala numérica de Dor permite Quantificar a intensidade da dor usando números de 0 a 10 . O ponto 0 (zero) representa nenhuma dor e 10 (dez) representa a pior dor possível ${ }^{(10)}$.

O Índice de Barthel ${ }^{(1)}$, utilizado para avaliação da capacidade funcional é composto por 10 atividades: alimentação, banho, higiene pessoal, vestir-se, intestinos, bexiga, transferência para higiene intíma, transferência - cadeira e cama, deambulação e subir escadas. Seu escore é obtido através da soma de todos os pontos obtidos, sendo considerado independente o individuo que atingir a pontuação total, isto é, 100 pontos. Pontuações abaixo de 50 indicam dependência em atividades de vida diária. Este instrumento é um dos mais utilizados para a avaliação da capacidade funcional em idosos, sendo que o Índice de Barthel tem demonstrado forte confiabilidade interexaminadores $(0,95)$ e confiabilidade teste-resteste $(0,89)$, assim como fortes correlações $(0,74$ a 0,80$)$ com outras medidas de incapacidade. Mediante esses dados optou-se neste estudo por utilizar o Índice de Barthel para alcançar ao objetivo proposto.

Esta pesQuisa foi aprovada pelo Comitê de Ética da Universidade Federal do Rio Grande do Norte/UFRN, obtendo parecer favorável para sua realização ( $n^{\circ}$ 177/05), obedecendo à Resolução 196/96 Que trata das pesquisas realizadas em seres humanos $^{(12)}$. Para participar da peseuisa voluntariamente o idoso ou 
seu responsável assinou o Termo de Consentimento Livre e Esclarecido.

As análises estatísticas foram realizadas pela aplicação do software SPSS versão 13.0. Para a caracterização da amostra do estudo realizou-se análise descritiva das variáveis com confecção de tabelas de freeuência, medidas de posição (média, mediana, mínima e máxima) e dispersão (desvio-padrão) visando caracterizar a amostra do estudo.

O Teste do Qui-Quadrado foi utilizado para verificar a associação entre a variável dependente (capacidade funcional) e as demais variáveis do estudo (Sexo, idade, renda, escolaridade, presença de doenças osteomusculares, tipos de doenças osteomusculares, presença de dor, intensidade de dor, tempo de instituciona-lização, localização da dor, intervenções na dor, intervenções na capacidade funcional e tempo de dor). Sendo adotado ainda o Teste Exato de Fischer Quando os valores absolutos nas distribuições das variáveis apresentavam valor inferior a 5 . O nível de significância adotado para os testes estatísticos foi de $5 \%$, ou seja, $p<$ ou $=0,05$.

\section{RESULTADOS}

Foram estudados 60 idosos institucionalizados, 50,0\% de cada sexo, sendo o sexo masculino mais freeuente na faixa etária de 60 a 75 anos (28,3\%). Com idade mínima de 60, máxima de 104 e média de 77,6 anos $( \pm 11,64)$. Quanto à escolaridade houve um maior predomínio de idosos analfabetos (73,3\%). Em relação à renda, 100\% dos idosos recebem um salário mínimo (R\$ 300,00 referente a dez/2005) provenientes da aposentadoria. No tocante ao tempo de institucionalização, 85,0\% moram no asilo no intervalo de 1 a 10 anos, sendo este período mais freeuente na faixa etária de 60 a 75 anos $(48,3 \%)$. As doenças osteomusculares estiveram presentes em $81,7 \%$ dos idoso apresentando destaque na faixa etária acima de 75 anos (41,7\%). As patologias mais citadas foram as articulares (artrite, artrose e artralgia) correspondendo a 49,9\% (Tabela 1).

A ocorrência de dor nos idosos foi de $73,3 \%$, sendo a sua distribuição igual entre as duas faixas etárias estudadas (36,7\%) res-pectivamente. Em relação à intensidade, 51,7\% dos idosos relataram dor intensa, com distribuição maior acima dos 75 anos $(31,7 \%)$. Quanto à localização da dor os idosos Queixaram-se mais dos MMII $(47,7 \%)$ e coluna (25,0\%), sendo mais frequente na faixa etária acima de 75 anos com 25,0\% e 13,6\% respectivamente (Tabela 2 ).

O tempo de dor variou de oito meses a 25 anos, com média de $4,05( \pm 4,65)$ meses. Na distribuição do tempo de ocorrência de dor em relação a faixa etária, foi mais frequente o tempo de dor referente a 1, 3, 4 e 5 anos com 13,6\% cada. Na faixa etária de 60 a 75 anos foi maior o tempo de dor correspondente a 3 anos $(9,1 \%)$ e acima de 75 anos o tempo de 1 ano $(6,8 \%)$. Em relação ao uso de intervenções no controle da dor apenas 15,9\% dos idosos recebem-na, sendo mais frequente na faixa etária acima de 80 anos $(9,1 \%)$ (Tabela 2).

Tabela 1. Variáveis sexo, escolaridade, renda, profissão, tempo de institucionalização, doenças osteomusculares, segundo faixa etária. Jequié, BA, 2008.

\begin{tabular}{|c|c|c|c|c|c|c|}
\hline \multirow{3}{*}{ Variáveis } & \multicolumn{5}{|c|}{ Faixa Etária } & \multirow{3}{*}{$\begin{array}{c}\text { Total } \\
\%\end{array}$} \\
\hline & \multicolumn{2}{|c|}{60 a 80} & \multicolumn{3}{|c|}{$>80$ anos } & \\
\hline & $\mathrm{n}$ & $\%$ & $\mathrm{n}$ & $\%$ & $\mathrm{n}$ & \\
\hline \multicolumn{7}{|l|}{ Sexo } \\
\hline Masculino & 20 & 33,3 & 10 & 16,7 & 30 & 50,0 \\
\hline Feminino & 15 & 25,0 & 15 & 25,0 & 30 & 50,0 \\
\hline \multicolumn{7}{|l|}{ Escolaridade } \\
\hline Não alfabetizado & 25 & 41,7 & 19 & 31,7 & 44 & 73,3 \\
\hline Alfabetizado & 8 & 13,3 & 6 & 10,0 & 14 & 23,3 \\
\hline Ensino fundamental & 1 & 1,7 & - & - & 1 & 1,7 \\
\hline Ensino superior & 1 & 1,7 & - & - & 1 & 1,7 \\
\hline \multicolumn{7}{|l|}{ Renda } \\
\hline $\begin{array}{l}\text { I Salário mínimo } \\
\text { Profissão }\end{array}$ & 35 & 58,3 & 25 & 41,7 & 60 & 100,0 \\
\hline Aposentado & 35 & 58,3 & 25 & 41,7 & 60 & 100,0 \\
\hline \multicolumn{7}{|l|}{ Tempo de Institucionalização } \\
\hline 1 a 10 anos & 31 & 51,7 & 20 & 33,3 & 51 & 85,0 \\
\hline$>10$ anos & 4 & 6,7 & 5 & 8,3 & 9 & 15,0 \\
\hline \multicolumn{7}{|l|}{ Doenças Osteomusculares } \\
\hline $\begin{array}{l}\text { Artrite/Artrose/Artralgia/ } \\
\text { Esporão do calcâneo/ } \\
\text { Osteoporose/ } \\
\text { Artrite reumatóide }\end{array}$ & 15 & 39,5 & 13 & 34,2 & 28 & 73,7 \\
\hline Hérnia de disco/ Lombalgia & 3 & 7,9 & 4 & 10,5 & 7 & 18,4 \\
\hline Tendinite/ Bursite & - & - & 3 & 7,9 & 3 & 7,9 \\
\hline
\end{tabular}

Tabela 2. Variáveis sexo, faixa etária, tempo de institucionalização e doenças osteomusculares, segundo a presença de dor. Jequié, BA, 2008.

\begin{tabular}{|c|c|c|c|c|c|c|c|}
\hline \multirow[b]{2}{*}{ Variáveis } & \multicolumn{6}{|c|}{ Presença de dor } & \multirow[b]{2}{*}{$p=$ valor } \\
\hline & $\mathrm{n}$ & $\%$ & $\mathrm{n}$ & $\%$ & $\mathrm{n}$ & $\%$ & \\
\hline \multicolumn{8}{|l|}{ Sexo } \\
\hline Masculino & 22 & 36,7 & 8 & 13,3 & 30 & 50,0 & \multirow[t]{2}{*}{1,000} \\
\hline Feminino & 22 & 36,7 & 8 & 13,3 & 30 & 50,0 & \\
\hline \multicolumn{8}{|l|}{ Faixa Etária } \\
\hline 60 a 75 anos & 22 & 36,7 & 10 & 16,7 & 32 & 53,3 & \multirow{2}{*}{0,599} \\
\hline Acima de 75 anos & 22 & 36,7 & 6 & 10,0 & 28 & 46,7 & \\
\hline \multicolumn{8}{|c|}{ Tempo de Institucionalização } \\
\hline 1 a 10 anos & 40 & 66,7 & 11 & 18,3 & 51 & 85,0 & \multirow{2}{*}{$0,048 \mathrm{f}$} \\
\hline$>10$ anos & 4 & 6,7 & 5 & 8,3 & 9 & 15,0 & \\
\hline \multicolumn{8}{|c|}{ Doenças Osteomusculares } \\
\hline Presente & 36 & 60,0 & 13 & 21,7 & 49 & 81,7 & \multirow{2}{*}{$0,104 f$} \\
\hline Ausente & 8 & 13,3 & 3 & 5,0 & 11 & 18,3 & \\
\hline
\end{tabular}


Na distribuição entre presença de dor com as variáveis do estudo (sexo, faixa etária, tempo de institucionalização e doenças osteomusculares) houve uma distribuição normal entre os sexos e faixa etária, correspondendo a $36,7 \%$ respectivamente. Verificou-se uma maior distribuição da dor entre os idosos não alfabetizados $(56,7 \%)$, com tempo de institucionalização entre 1 a 10 anos $(66,7 \%)$ e Que apresentavam doenças osteomusculares (60,0\%). Foi encontrada diferença estatísticamente significante apenas entre a presença de dor e tempo de institucionalização $(p=0,048)$, com a realização do Teste Qui-Quadrado (Tabela 2).

Na distribuição entre os tipos de doenças osteomusculares (articulares e musculares) com a presença de dor houve uma maior frequência de doenças articulares $(53,1 \%)$ e verificou-se no Teste Qui-Quadrado uma diferença estatisticamente significante $(p=0,045)$.

A pontuação do Índice de Barthel variou de 10 a 100 pontos, com média de $69,75( \pm 26,5)$ pontos, na distribuição com as variáveis do estudo os idosos foram classificados como dependentes nas variáveis, sexo feminino $(38,3 \%)$, faixa etária acima de 75 anos (36,7\%), com nível de escolaridade não alfabetizado $(53,3 \%)$, tempo de institucionalização entre I a 10 anos $(61,7 \%)$, presença de doenças osteomusculares $(55,0 \%)$ e doenças articulares $(49,0 \%)$. Nessa distribuição não foi encontrada diferença estatísticamente significante Quando submetidos ao Teste Qui-Quadrado.

Na pontuação do Índice de Barthel, 70\% dos idosos foram classificados como dependentes. Sendo ainda classificados como dependentes na faixa etária entre 60 a 75 anos em duas atividades transferência cama e cadeira $(33,3 \%)$ e deambulação $(28,3 \%)$. Na faixa etária acima de 75 anos em cinco atividades subir escadas $(33,3 \%)$, transferência cama e cadeira $(33,3 \%)$, transferência para a higiene intíma $(26,7 \%)$, vestir-se $(26,7 \%)$ e deambulação $(25,0 \%)$ (Tabela 3).

Com a aplicação do Teste Qui-Quadrado verificou-se diferença estatisticamente significante entre presença de dor e as atividades: banho $(p=0,015)$ vestir-se $(p=0,041)$, transferência para higiene intima $(p=0,001)$, transferência cama e cadeira $(p=0,032)$, deambulação $(p=0,010)$ e subir escadas $(p=0,008)$. Sendo ainda encontrada diferença estatisticamente significante entre a pontuação total de Barthel (dependente/independente) e presença de dor, $\mathrm{p}<0,000$.

Em relação à utilização de intervenções para a preservação e/ ou manutenção da capacidade funcional apenas 18,3\% utilizam tais intervenções, sendo a fisioterapia a única medida utilizada.

\section{DISCUSSÃO}

O presente estudo avaliou a influência da dor na capacidade funcional de idosos institucionalizados e envolveu 60 idosos. A faixa etária mais frequente foi entre 60 e 75 anos (53,3\%) sendo $28,3 \%$ dos idosos do sexo masculino. A distribuição entre as faixas etárias está de acordo com a realidade nacional, mas a distribuição Quanto ao sexo não reflete a realidade do Brasil, visto Que a mulher figura em maior número na faixa etária acima de $60 \mathrm{anos}^{(2)}$. A predominância de idosos pertencentes ao sexo feminino tem sido atribuída à menor exposição a determinados fatores de risco, notadamente no trabalho, menor prevalência de tabagismo e uso de álcool, diferença Quanto à atitude em relação a doenças e incapacidades e, por último uma maior cobertura de assistência gineco-obstetrica $^{(13-14)}$.

Quanto ao índice de escolaridade, 73,3\% dos idosos são anal- 
fabetos. Os alfabetizados totalizam $26,7 \%$, fato Que caracteriza o baixo índice de escolaridade. Em uma pesquisa realizada no Nordeste do Brasil constataram-se taxas elevadas de analfabetismo entre os idosos, aproximadamente 65 em cada 100 idosos não sabiam ler e escrever ${ }^{(15)}$. Em relação à renda, verificou-se Que $100 \%$ dos idosos apresentavam aposentadoria de 1 salário mínimo. Em estudo realizado no Nordeste do Brasil, verificou-se que 50\% dos idosos com 80 anos ou mais tinham renda até meio salário mínimo, ao passo que na faixa entre 60 a 69 anos este percentual foi de cerca de $35 \%{ }^{(15)}$.

Nesse estudo foram identificadas apenas as doenças osteomusculares, estando estas presentes em 81,7\% dos idosos e sendo a artrite, artrose e artralgia. Dados estes corroborados por um estudo realizado com 375 idosos institucionalizados sobre Queixa álgica observou-se uma prevalência de $49 \%$ de casos de artrite ${ }^{(17)}$. A idade avançada é um importante fator de risco para a ocorrência de patologias osteomusculares, uma vez Que com o processo de envelhecimento biológico ocorre comprometimento das estruturas do aparelho locomotor sendo afetados os ossos, músculos, tendões e articulações de todas as regiões do corpo ${ }^{(17)}$.

No presente estudo observou-se em 73,3\% dos idosos a presença de dor. Esses achados assemelham-se aos encontrados em pesQuisas nacionais, visto Que em estudo sobre dor em idosos asilados relataram prevalência Que variou de $71 \%$ a $83 \%^{(18-19)}$. A dor compromete gravemente o desempenho do doente geriátrico, resulta em menor capacidade para executar as atividades de vida diária e compromete a Qualidade de vida diária ${ }^{(20-21)}$.

A dor crônica na terceira idade pode apresentar importantes complicações tais como depressão, ansiedade, isolamento social, distúrbios do sono, agitação, agressividade, comprometimento da função cognitiva, incapacidade funcional e diminuição da Qualidade de vida, levando a dependência em atividades de vida diária e a um maior gasto com serviços sociais ${ }^{(20)}$.

Nesse estudo, as localizações nas Quais a presença de dor predominaram foram membros inferiores $(47,7 \%)$ e coluna $(25,0 \%)$. A associação de dor em membros inferiores e coluna foram encontradas em estudos epidemiológicos de dor musculoesquelético no qual foram estudadas 258 pessoas de 60 a 74 anos buscando avaliar a relação de dor em Quadril e joelho com outras condições mórbidas e incapacidade ${ }^{(6-22)}$. Entre os 124 com morbidade associadas, 10,98\% tinham dor em Quadril e joelho e dor lombar. Estudos evidenciam Que os MMII são os segmentos corporais mais acometidos por dor, isto devido a degeneração cartilaginosa decorrente do envelhecimento biológico ou da ocorrência de patologias crônico-degenerativas a exemplo da artrite e artrose ${ }^{(6)}$.

A dor foi caracterizada ainda Quanto à freQuência, duração e intensidade dos episódios. No presente estudo a dor foi classificada mais frequentemente como dor intensa. Semelhante ao encontrado nesse estudo, em estudo dois estudos sobre dores em geral, realizados com idosos institucionalizados, encontrou-se ente 18\% e $52 \%$ dos idosos com dor intensa ${ }^{(6-23)}$. Um estudo com 100 idosos institucionalizados encontrou $66 \%$ deles com dores intermitentes sendo Que $51 \%$ dessas dores ocorriam diariamente e 35\% semanalmente ${ }^{(6-18)}$.

Há muitas evidências de Que a dor em idosos continua a ser subavaliada, subescrita e conseQuentemente subtratada, neste estudo apesar da dor estar presente em 73,3\% dos idosos, apenas, apenas $15,9 \%$ realizava tratamento medicamentoso para o alívio da dor. As consequências da dor mal controlada incluem comprometimento da deambulação, redução da socialização, diminuição da independência nas atividades da vida diária e redução global da Qualidade de vida ${ }^{(16,18)}$.

As incapacidades física, psicológica e social são as principais repercussões Que comprometem a Qualidade de vida dos idosos com dor. A avaliação da capacidade funcional na gerontologia é importante indicativo da Qualidade de vida do idoso, sendo o desempenho nas atividades da vida diária o parâmetro amplamente aceito e reconhecido, pois permite aos profissionais de saúde e, em particular ao Fisioterapeuta uma visão mais precisa Quanto à severidade da doença e das suas sequelas ${ }^{(24)}$.

Nesse estudo a maioria dos idosos foi denominada como dependentes, sendo os idosos classificados como dependentes em Quatro atividades (transferência para higiene íntima, transferência cama e cadeira, deambulação e subir escadas). As dificuldades progressivas na realização das atividades funcionais básicas aumentam com a idade e, freQüentemente, decorrem das alterações fisiológicas do processo de envelhecimento, de patologias e/ou de problemas associados a essa faixa etária. Segundo a Organização Mundial de Saúde, após os 70 anos, 30\% dos idosos são portadores de alguma patologia crônica e mais: entre os portadores desse tipo de patologia, cerca de $50 \%$ tem algum tipo de limitação ou incapacidade física ${ }^{(25)}$.

Em estudo realizado no município de João Pessoa com 30 idosos na faixa etária de 62 a 93 anos, todos apresentavam dependência na realização das atividade básicas de vida diária ${ }^{(26)}$. Resultados semelhantes aos encontrados neste estudo foram encontrados em estudo realizado no interior do Rio Grande do Sul, no Qual os idosos asilados foram classificados como dependentes nas atividades de banho $(67,89 \%)$, vestuário $(53,22 \%)$, higiene pessoal $(53,21 \%)$, alimentação $(57,8 \%)$ e bexiga $(65,13 \%)^{(27)}$.

A perda da capacidade funcional estar associada a predisposição de fragilidade, risco aumentado de Quedas e morte, aumento de Quedas da mobilidade, dependência e institucionalização gerando cuidados de alto custo e longa permanência ${ }^{(17,22)}$. A manutenção da capacidade funcional é importante fator para a independência e Qualidade de vida dos idosos. No entanto a capacidade funcional pode ser limitada pela presença de dor. Estudos sugerem Que a dor no idoso interfere na capacidade funcional, visto Que há relação entre presença de dor e a realização de atividades de vida diária ${ }^{(26)}$.

Em estudo realizado em Belo Horizonte com 35 idosos verificou-se que a intensidade da dor está relacionada ao comprometimento da capacidade funcional. Dados estes corroborados por este estudo em Que foi encontrada diferença estatística significativa entre dor e capacidade funcional ${ }^{(28)}$. PesQuisas sugerem Que a dor no idoso interfere na independência e conseqüentemente na Qualidade de vida, uma vez Que pode haver correlação entre dor e incapacidade para a realização das atividades de vida diária. Considerações funcionais especiais incluem o reconhecimento de Que atividades avançadas e eletivas da vida diária podem ser mais sensíveis às alterações dolorosas, desta forma comprometendo a capacidade funcional.

Sob influência da dor, o idoso poderá apresentar uma progressiva reclusão social, com tendência ao sedentarismo, perda de autoestima e abandono de autocuidados. Nesse momento a capacidade 
funcional encontra-se bastante comprometida, podendo haver dependência física e mental para a realização de atividades da vida diárias mais complexas, como por exemplo, limpar a casa, fazer compras, cuidar das finanças.

Estes conhecimentos acerca da influência da dor na capacidade funcional de idosos institucionalizados a partir da utilização dos instrumentos destinados a avaliação da capacidade funcional e da dor na terceira idade, são relevantes para atuação dos profissionais de saúde junto a esta parcela crescente da população, a fim de proporcionar a estes uma maior autonomia e independência com consequente melhoria da Qualidade de vida.

Mediante as precárias condições de saúde prestadas ao idoso na instituição asilar por falta de recursos humanos e materiais, visto Que se trata de uma instituição filantrópica Que sobrevive de doações, se faz necessário, portanto, Que sejam disponibilizados aparatos infra-estruturais, tanto em recursos humanos Quanto materiais, para atender, corretamente a essa clientela, levando se em consideração as particularidades dessa faixa etária e a especificidade de cada um.

Diante da realidade estudada em Que verificou-se uma alta prevalência de dor e um elevado número de idosos com comprometi- mento da capacidade funcional, fatores estes Que comprometem a Qualidade de vida do idoso, considera-se necessária a atenção e Qualificação de recursos humanos voltados, especificamente, ao atendimento dos idosos ${ }^{(29)}$. Outrossim, destaca-se ainda a necessidade de incentivo governamental, tanto em forma de verbas e isenção de impostos, Quanto na criação de serviços de atenção terciária ao idosos, os Quais Ihe possibilitem, ao menos, continuar sob o convívio familiar, o Que, certamente, representa menores custos de atendimento dessa população.

\section{CONCLUSÕES}

Constatou-se nesse estudo uma elevada prevalência de dor intensa entre os idosos institucionalizados e localização nos membros inferiores. Em relação à capacidade funcional a maioria dos idosos institucionalizados foi classificada como dependentes conforme o Índice de Barthel. Ao averiguar a influência da dor sobre a capacidade funcional dos idosos institucionalizados encontrouse uma parcela si-gnificativa dos idosos com dor apresentando dependência na reali-zação das atividades de vida diária, sendo encontrada diferença estatística significante $(p=0,000)$.

\section{REFERÊNCIAS}

I. Tavares MF, Júnior RCF. A promoção da saúde nas instituições de longa permanência: uma reflexão sobre o processo de envelhecimento no Brasil. Rev Bras Geriatr Gerontol 2006; 9 ( I): 17-30.

2. Silva ANA, Santa Clara NS. Perfil dos idosos institucionalizados e prevalência de dependência em uma instituição de longa permanência da cidade de Caratinga - MG. [monografia]. Caratinga: Faculdade de Fisioterapia de Caratinga, FUNEC; 2003.

3. Davim RMB, Torres GV, Dantas SMM, Lima VM. Estudo com idosos de instituições asilares no município de Natal/ RN: Características socioeconômicas e de saúde. Rev Latino-am Enfermagem 2004; 12(3): 5 18-24.

4. Silvestre IA, Costa NMM. Abordagem do idoso em programas de saúde familiar. Cad Saúde Pública 2003; 19(3): 733-43.

5. Amero P, Muriel C, Santos JFI, Rodrigues RE, Gonzalez RS. Bases genéticas del dolor. Rev Soc Espanhola Dolor 2004; I I (7): 444-5 I.

6. Barro N. Manifestações clínicas da dor crônica e princípios de tratamento. Dor Diagn Trat 2004; I (3): 22-7.

7. Hiroshi S, Sugisawa H, Shuichiro W. Functional capacity in elderly: The role of chronic diseases and dementia. Cross-sectional and longitudinal data from a population-based study. Ger Gerontol Int 2001; 1: 8-13.

8. Raso V. A adiposidade corporal e a idade prejudicam a capacidade funcional para realizar as atividades da vida diária de mulheres acima de 47 anos. Rev Bras Med Esporte 2002; 8(6): 19- 23.

9. Wang L, Van Belle G, Kukull W, Larson E. Predictors of functional change: a longitudinal study of nondements people aged 65 and older. I Am Geriartr Soc 2002; 50: 1525-34.

10. Andrade FA, Pereira LV, Sousa FAEF. Mensuração da dor no idoso: uma revisão. Rev Latino-am Enfermagem 2006; I4(2): 271-6.

I I. Pimenta CAM, Teixeira MI. Questionário de dor de McGill: proposta de adaptação para a Língua portuguesa. Rev Bras

Anestesiol 1997: 47 (92): 177-87.

12. Ricci NA, Kubota MT, Cordeiro RC. Concordãncia de observações sobre a capacidade funcional de idosos em assistência domiciliar. Rev Saúde Pública 2005; 39(4): 53-9.

13. Garcia RA, Carvalho IAM. O envelhecimento da população brasileira: um enfoque demográfico. Cad Saúde Pública 2003; 19(3): 725-33.

14. Giatti L, Barreto SM, Lima MFC. Condições de saúde, capacidade funcional, uso de serviços de saúde e gastos com medicamentos da população idosa brasileira: um estudo descritivo baseado na pesquisa Nacional por Amostra de domicílios. Cad Saúde Pública 2003; 19(3); 735-43.

15. Ramos LR, Coelho IM. Epidemiologia do envelhecimento no Nordeste do Brasil: resultados de um ineuérito domiciliar. Rev Saúde Pública 1999; 33(5): 1-8.

16. Parmelle PA, Smith B, Katz IR. Pain complaents and cognitive status among elderly instition residents. I Am Geriatr Soc 1993; 41: 517-22.

17. Reis LA, Mascarenhas CHM, Torres GV. Evaluation of functional capacity on institutionalized elderly in the City of JeQuié/ BA. Fiep Bulletin 2008; 78(1): 10-5.

18. Teixeira MJ. Fisiopatologia da dor. Rev Med São Paulo 1997; 76 (1): 7-20.

19. Augusto ACC, Soares CPSS, Resende MA, Pereira LSM. Avaliação da dor em idosos com doença de Alzheimer: uma revisão bibliográfica. Textos Envelhec 2004; 7 (1): 2 I-5.

20. Helme RD, Gibson SJ. Pain in the elderley. In: Jensen TS, Turner JA, Wesenfeld ZH, editors. Proceedings of the VIII World Congress on Pain: Progress in Pain Research and Management. Seattle, IASP Press 1997; 8: 919-44.

21. Ferrell BA. Pain management in elderly people. I Am Geratr Soc 1991; 39: 64-73.

22. Reis LA, Torres GV, Reis LA. Características sócio-demográficas e de saúde de idosos de uma instituição do município de 
Jequié/BA. Rev Espaço Saúde 2008; 9(1): 3 I-45.

23. Magni G, Marchetti M, Moreschi C. Chronic musculoskeletal pain an depressive symptoms in the National Health and Nutrition Examination: epidemiologic follow-up study. Pain 1993; 53-163-8

24. Guimarães LHCT, Galdino DCA, Martins FLMM, Abreu SR, Lima M, Vitoriano DFM. Avaliação da capacidade funcional de idosos em tratamento fisioterapêutico. Rev Neurocien 2004; 12(3): 22-9.

25. Pizzolli SML, Ruete REN, Gagel CA, Santos MD. Avaliação gerontologica através do Índice de Katz (nível de dependência) no Lar de idosos de Paragaçu Paulista. Omnia Saúde 2006; I(I): 26-3I.
26. Fernandes MGM, Garcia TR. Determinantes da tensão do cuidador familiar de idosos dependentes. Rev Bras Enferm 2009; 62(1): 57-63.

27. Silveira RCR, Guedes JM. Análise da capacidade funcional da população geritrica institucionalizada na cidade de Passo Fundo-RS. Rev Bras Ciên Envelhec Humano 2004; 10-2 I .

28. Ribeiro RC, Marin HF. Proposta de um atendimento de avaliação da saúde do idoso institucionalizado baseado no conceito do conjunto de dados essenciais em enfermagem. Rev Bras Enferm 2009; 62(2): 204-12. 